\title{
Declaración de la Academia Chilena de Medicina sobre el Título V de la Ley 20.850 (Ley Ricarte Soto) y su proyecto de reglamento "De los ensayos clínicos de productos farmacéuticos y elementos de uso médico"\#
}

\author{
Comité de Estudio y Redacción: GLORIA LÓPEZ S.****, GLORIA VALDÉS S.**,****, \\ EMILIO ROESSLER B. ${ }^{* * * * * * * *}$ y VICENTE VALDIVIESO D.**,****
}

\author{
Declaration of the Chilean Academy of Medicine of Law 20.850 \\ "On clinical trials of pharmaceutical products and medical devices" \\ and of the bylaw that will regulate its application
}

In Chile, high cost treatments required by selected medical conditions are financed by the State, according to Law 20.850. A bylaw under discussion by the Senate regulates clinical trials, posing complex issues that will endanger local interest in front-line research: 1. The exclusive and mandatory control bestowed to the Institute of Public Health during all stages of the trials and also the surveillance of institutions performing clinical trials, overriding their Clinical Research Review Boards; 2.The 10 years period during which any adverse event is assumed to have been caused by the medication or device evaluated by the trial, unless the contrary is proven in a judicial process; 3. Individuals submitted to the trials are entitled to free post trial access to the treatment received during the study, financed by the trial supporting entities and as long as the drug or device is considered to be useful. While agreeing with the need to have a National Registry of Clinical Trials, we predict that the mentioned critical issues in the bylaw will lead to difficulties and unnecessary judicial processes, thus limiting clinicians'interest in performing research. We propose to modify the bylaw, excluding responsibilities on events associated with the natural evolution of the medical condition, or with patients'ageing, or with comorbidities and clinical events considered unpredictable when the protocol was accepted. We recommend that the free post trial access should be a joint decision involving the patient and the attending physician, taking in consideration that the volunteer has been exposed to risks and burdens, or when discontinuation of treatment entails a vital risk until the treatment under study has been approved and becomes available in the national market.

Key words: Clinical trials as topic; Clinical trials data monitoring committees; Drugs, investigational; Jurisprudence; Public Health.

Documento aprobado por unanimidad, por Miembros de la Academia, el 22 de diciembre de 2016.

\#Este documento se ha publicado simultáneamente en Revista Médica de Chile, Revista Chilena de Enfermedades Respiratorias y Revista Chilena de Infectología por la relevancia de su contenido y la conveniencia de ampliar su acceso a los lectores.

\footnotetext{
* Miembro de la Academia y Presidenta del Comité.

** Miembros de la Academia.

*** Facultad de Medicina, Campus Norte, Universidad de Chile.

**** Facultad de Medicina, Pontificia Universidad Católica de Chile.

***** Facultad de Medicina, Clínica Alemana de Santiago-Universidad del Desarrollo.
} 


\section{Resumen}

En Chile los tratamientos de alto costo requeridos por seleccionadas condiciones médicas son financiados por el Estado, de acuerdo a la Ley 20.850, que se hizo efectiva desde noviembre de 2015. Un reglamento de esta ley-actualmente en discusión por el Senado-incluye la regulación de los ensayos clínicos y plantea importantes aspectos que van a poner en riesgo la realización de investigaciones clínicas avanzadas: 1. El control exclusivo y mandatorio otorgado al Instituto de Salud Pública durante todas las etapas de los ensayos y la vigilancia de las instituciones que los realizan, que sobrepasa las atribuciones de los Comités de Ética Cientifica Institucionales; 2. El periodo de hasta 10 años después de la aparición de cualquier efecto adverso, durante el cual se asume causado por el medicamento o dispositivo evaluado en el ensayo, mientras no se demuestre lo contrario en un proceso judicial; 3. Los participantes de los estudios tienen derecho a continuar con el tratamiento recibido durante el estudio una vez terminado este, financiado por las entidades que patrocinan los estudios y mientras el fármaco o dispositivo se considere útil. Estamos de acuerdo con la necesidad de contar con un Registro Nacional de Ensayos Clínicos. Sin embargo, predecimos que los aspectos críticos del reglamento causarán dificultades y procesos judiciales innecesarios, lo que limitará el interés de los clínicos en realizar investigación. Proponemos que el reglamento debe modificarse a fin de excluir responsabilidades sobre eventos asociados con la evolución natural de la condición clínica, el envejecimiento del paciente, comorbilidades y con eventos clínicos no predecibles cuando se aceptó el estudio. Recomendamos que el acceso gratuito posterior al estudio debe constituir una decisión conjunta del paciente y su médico tratante, considerando los riesgos y la carga a que se expuso el paciente, o al riesgo vital secundario a la suspensión del tratamiento del estudio mientras no esté disponible en el mercado nacional.

Palabras clave: Ensayos clínicos como tópico; Comités de monitoreo de datos de ensayos clínicos; Drogas de investigación; Jurisprudencia; Salud Pública.

\section{Ensayos clínicos: definición y niveles}

El centro de la Medicina es el enfermo y su principio rector es buscar siempre su bien; por lo tanto, la investigación biomédica debe beneficiarlo, protegerlo y no dañarlo. Para progresar en la prevención, el diagnóstico y el manejo de las enfermedades con elementos nuevos o más eficaces son indispensables herramientas cuya utilidad y riesgo deben probarse mediante el método científico aplicado a la investigación clínica. Esta investigación representa un paso en el continuo de la investigación "traslacional", que va progresivamente desde la investigación básica realizada en el laboratorio en animales u otros modelos, a diversas etapas de estudios clínicos en humanos, para finalmente ser incorporados al uso generalizado en pacientes ${ }^{1}$.

Dentro de los estudios clínicos, los "ensayos clínicos" tienen un rol importante, por lo que es indispensable definirlos. Con este propósito citamos la definición dada por el International Committee of Medical Journal Editors o $I C M J E^{2}$, que ha sido adoptada universalmente: "El ICMJE define como ensayo clínico a todo proyecto de investigación que asigne prospec- tivamente a una persona o grupos de personas a una intervención, con o sin su comparación concurrente o grupos controles, para estudiar la relación causa-efecto entre una intervención relacionada con salud y un resultado o efecto en salud. Las intervenciones relacionadas con salud son aquellas utilizadas para modificar un resultado biomédico o relacionado con salud; sus ejemplos incluyen fármacos, procedimientos quirúrgicos, dispositivos, tratamientos conductuales, intervenciones dietéticas, intervenciones para mejorar la calidad de la salud y de los cuidados de la salud. Los resultados en salud son cualquier medición biomédica o relacionada con salud obtenida en pacientes o participantes, incluyendo mediciones fármaco cinéticas y eventos adversos" 3 .

Con el fin de analizar el Título V de la Ley 20.850 "De los ensayos clínicos de productos farmacéuticos y elementos de uso médico", nos referiremos a los ensayos clínicos de fármacos y dispositivos habitualmente realizados a iniciativa de, y con el respaldo financiero de, empresas farmacéuticas y fabricantes de equipos médicos, en múltiples centros internacionales, con tratamientos asignados a personas que se 
adscribieron voluntariamente al ensayo mediante un consentimiento informado, después de recibir información completa sobre la naturaleza y el desarrollo de ese ensayo

La investigación científica que estudia mecanismos fisiopatológicos en seres humanos no representa un ensayo clínico. Tampoco son ensayos clínicos las investigaciones epidemiológicas que no involucren una intervención explícita e inmediata en los cuidados de la salud de las personas estudiadas.

Los ensayos clínicos tienen diferentes niveles. Los más simples repiten estudios anteriores de fármacos o de dispositivos ya aprobados y que se usan en otros países, con el propósito de evaluar la respuesta propia de nuestra población; estos ensayos suelen incluir tácticas de mercado de los fabricantes del producto ensayado; lo mismo ocurre con el estudio de nuevos usos de fármacos aprobados y con seguridad demostrada. Los ensayos de complejidad intermedia están dirigidos a evaluar fármacos o dispositivos que son una modificación química, biológica o técnica de otro elemento "madre" ya conocido, o porque son empleados en otro contexto clínico o en dosis distintas (generalmente mayores) a las previamente evaluadas, y requieren declarar con prontitud los efectos adversos detectados. Por último, los más complejos son los ensayos que evalúan fármacos o dispositivos absolutamente innovadores que, de ser exitosos, llenan una necesidad real y para la cual la medicina no tenía una herramienta eficaz; estos ensayos obligan a una mayor vigilancia de los voluntarios y a considerar continuar con la intervención después del término del ensayo.

\section{Ensayos clínicos en la base conceptual de la Ley 20.850 (Ley Ricarte Soto)}

El Ministerio de Salud y los legisladores que participaron en la redacción y aprobación de la Ley 20.850 consideraron justamente la importancia que tienen las evidencias provistas por los ensayos clínicos (artículo $7^{\circ}$ ): “(en) el proceso destinado a determinar los diagnósticos y tratamientos de alto costo con Sistema de Protección Financiera se realizará una evaluación científica basada en la evidencia disponible, para el diagnóstico o tratamiento de una condición específica de salud". La literatura médica registra, precisamente, numerosos ensayos clínicos dirigidos a comprobar el beneficio de medicamentos de alto costo para la mayoría de las patologías seleccionadas en la Ley $20.850^{4-22}$.

\section{Comentarios a los principales problemas detectados en el Título $V$ "De los ensayos clínicos de productos farmacéuticos y elementos de uso médico" y en el proyecto de reglamento que regulará las materias de las que trata dicho documento}

Paradojalmente, a pesar del valor que se reconoce en la Ley a la evidencia proporcionada por los ensayos clínicos, después de analizar los artículos correspondientes de la Ley y la propuesta de Reglamento, estimamos pertinente señalar, en primer lugar, que el objetivo de legislar para mejorar y regular la investigación médica y proteger a las personas sujetos de las investigaciones, no se cumple en debida concordancia con lo que establece su artículo $7^{\circ}$. La tarea habría sido más eficiente si la investigación médica se hubiese abordado globalmente y en forma separada, considerando los complejos aspectos propios de una actividad esencial para lograr progresos en la salud de la población.

Consideramos que la Ley que entró en vigencia en noviembre de 2015, y la propuesta de Reglamento que se discute actualmente en el Ministerio de Salud, introducen dificultades para realización de ensayos clínicos de fármacos y dispositivos médicos. Pensamos que conlleva riesgos de judicialización y deterioro de la relación médico paciente. Estas cortapisas y riesgos son difícilmente superables dado que los principales problemas de la Ley no pueden modificarse con rapidez, mientras son refrendados e iterados en distintos artículos del Reglamento propuesto, donde sí podrían introducirse cambios que eviten o disminuyan sus riesgos.

Queremos destacar sus puntos más controversiales:

1) El rol fiscalizador absoluto que se asigna al Instituto de Salud Pública (ISP) en las distintas etapas de la investigación clínica (mencionada como tal y sin circunscribirla explícitamente a los ensayos clínicos) y sobre los distintos organismos que participan en ella;

2) La continuación de la entrega del fármaco en estudio una vez terminado el protocolo de investigación, $\mathrm{y}$

3) El prolongado período de 10 años contados desde la manifestación del daño, durante el que se presume que cualquier efecto adverso en un sujeto que participó en un ensayo clínico es secundario al tratamiento aplicado, hasta que no se pruebe lo contrario. 


\section{1) Rol del ISP en las distintas etapas de un ensayo clínico}

Partimos por reconocer la necesidad de que los centros en que se realizan ensayos clínicos sean acreditados "por el Instituto de Salud Pública conforme a los estándares, exigencias y procedimientos que se indican a continuación" (artículo 46).

Siguiendo normas dictadas por la Organización Mundial de la Salud, es necesario implementar en Chile un Registro Nacional de Ensayos Clínicos, centrado en el ISP y con acceso universal, en el cual deben registrarse todos los ensayos clínicos que se realicen en el país, sea con patrocinio de corporaciones transnacionales o nacionales ${ }^{2,25}$.

Sin embargo, tenemos la convicción de que, para que el ISP apruebe y registre el ensayo de un fármaco o de un dispositivo, deben ser los Comités de Ética de Investigación respectivos quienes hayan analizado previamente el protocolo de la investigación, definido el tipo de estudio clínico que se empleará, su originalidad y las medidas que se tomarán para salvaguardar los intereses de los enfermos. Estos Comités están constituidos -entre otros miembros- por expertos en investigación y en bioética, y tienen la responsabilidad de controlar el seguimiento de los ensayos clínicos. La revisión de los protocolos de investigación por miembros designados por el Presidente del Comité y su análisis en sesión ampliada por los distintos especialistas del mismo, permiten emitir una resolución consensuada y fundamentada. Además, cada Comité y el Director de la institución huésped del ensayo clínico han sido sometidos a un riguroso proceso de acreditación y conocen el ambiente humano y los recursos materiales del sitio en que se desarrollaría el ensayo.

Con respecto al rol del ISP, estableciendo para los ensayos clínicos "su exclusivo y obligatorio control, fiscalización y vigilancia por el Instituto de Salud Pública", es necesario recordar que en Chile la mayoría de estos centros han sido formados en el seno de Facultades de Medicina y se mantienen estrechamente ligados a ellas, por lo que puede limitarse y entorpecerse la autonomía universitaria. En nuestra opinión, toda otra investigación médica que no se ajuste a la definición de "ensayo clínico" deberá autorizarse y controlarse solamente por las corporaciones (ej.: universidades) y las autoridades de salud locales, salvo cuando requiera el uso de un fármaco o dispositivo no aprobado aún por el ISP.

En cuanto a la disposición que establece que "el Director del Centro de Investigación
Clínica no podrá ser a la vez investigador responsable de un protocolo que se ejecute en su establecimiento" nos parece inconveniente que el Director mencionado no pueda participar en ensayos clínicos mientras dure en su cargo, ya que esta experiencia forma parte de sus antecedentes curriculares, valiosos para su selección y nombramiento. Estas personas no son numerosas en nuestro país y parece inconveniente que por asumir un cargo administrativo se les obligue a abandonar las actividades que garantizan que lo ejercerá con experiencia y calidad; además, esta restricción dificulta que acepten la dirección de un centro de investigación clínica. La declaración de conflictos de intereses y la exclusión de su persona al evaluarse sus propios proyectos de investigación evita sesgos en el proceso de revisión de ellos.

Respecto a la "suspensión o cancelación de la acreditación por oficio o por denuncia de terceros" (artículos 73 y 74), en un campo basado en la confidencialidad por sus evidentes implicaciones clínicas, farmacológicas y económicas, consideramos que se presta a denuncias sin fundamento real, y no parece prudente favorecer su génesis, dando motivo a la suspensión e incluso la cancelación de un centro que demanda un gran esfuerzo crear y mantener. Este mecanismo puede generar graves dificultades al ISP, obligando a investigaciones administrativas con fundamento escaso o nulo, que significarán pérdidas de tiempo y recursos e incluso conducirán a una judicialización paralizante.

\section{2) Mantención del tratamiento post ensayo}

Sin duda que es necesario velar por el cuidado del paciente que ha participado en un ensayo clínico y se ha beneficiado de un fármaco, especialmente si la suspensión de este tratamiento conlleva un riesgo vital. La declaración de Helsinki (2013) al estipular los requerimientos post ensayos, señala en su artículo 34 que "antes del ensayo clínico, los auspiciadores, investigadores y los gobiernos de los pacientes anfitriones deben proveer el acceso post ensayo a todos los participantes que todavía necesitan una intervención que ha sido identificada como beneficiosa en el ensayo, información que se debe proporcionar a los participantes durante el proceso del consentimiento informado". La continuación del tratamiento empleado en el estudio, o Post Trial Access (PTA), es una opción atractiva que se basa en el principio de reciprocidad: de la compañía farmacéutica o el fabricante que se benefician con la aprobación del nuevo fármaco o dispositivo, de 
los investigadores que ganan experiencia y visibilidad, y de la comunidad que potencialmente recibirá el fármaco cuando esté disponible para uso clínico. Sin embargo, el voluntario sometido al ensayo también obtiene beneficios de su participación y son varios los obstáculos para continuar con el nuevo tratamiento. La única instancia en que sería plausible la obligación contractual de continuar con el tratamiento es si el voluntario ha estado expuesto a riesgos o cargas importantes y sin PTA no tendrá la posibilidad del beneficio que se le debe por su participación, particularmente si la suspensión del fármaco en estudio lo pone en riesgo vital ante la carencia de alternativas terapéuticas ${ }^{23}$. Esta es la situación predominante en diferentes países y la duración de la entrega del tratamiento está fijada hasta la aprobación por la entidad correspondiente y su disponibilidad en el mercado ${ }^{24}$.

Concordamos con esta posición, ya que en ensayos clínicos con fármacos innovadores $\mathrm{y}$ en patologías para las cuales no se dispone de tratamientos eficaces, si se obtienen resultados favorables evidentes la ética impone el acceso al nuevo fármaco a todos los pacientes, incluidos los del grupo placebo. Es habitual que estos escasos estudios sean finalizados anticipadamente, por la evidente diferencia de resultados entre las ramas. En esas circunstancias es cuando se debe asegurar la disponibilidad del tratamiento, mientras mantenga su eficacia. Las vías deberán ser a costa del patrocinador o titular del registro transitorio, hasta que se obtenga el registro definitivo por el ISP y el tratamiento se incorpore a las Guías Clínicas. En la mayoría de los ensayos clínicos, los resultados son menos categóricos y resulta complejo prolongar un tratamiento más allá del término del ensayo en pacientes individuales debido a la latencia entre el término del estudio, la apertura de los datos del grupo a que pertenecen los pacientes y su análisis. No es fácil distinguir el beneficio objetivo de una intervención con la mejoría de la calidad de vida dada exclusivamente por la frecuencia de controles, exámenes de laboratorio y la seguridad de contar con un acceso permanente al equipo de estudio.

$\mathrm{Si}$ el paciente considera que fue beneficiado por el ensayo clínico podría exigir continuar en el protocolo de estudio, bajo la cobertura de un seguro, hasta el momento que él decida. Por lo tanto, se necesita una definición de utilidad más objetiva y fácil de determinar.

Sugerimos que la decisión de continuar la terapia debe hacerse caso a caso una vez conocidos los resultados finales del ensayo, incluidos los de seguridad. Es una decisión clínica que tomarán el paciente (que dejó de ser sujeto de estudio) y su médico tratante. A nuestro juicio no es adecuado que sea una medida universal para todos los individuos que participaron en los ensayos clínicos, aunque los resultados sean favorables en las condiciones del ensayo, puesto que los individuos no necesariamente presentan la misma magnitud de beneficios y riegos calculada para el análisis estadístico global.

\section{3) Prolongada responsabilidad de daños presumiblemente secundarios al estudio}

La presunción de que un daño acreditado en un voluntario que ha participado en un ensayo clínico, como se señala en el artículo 83 "si estos hechos o circunstancias que no se hubieran podido prever o evitar según el estado de los conocimientos" "existentes en el momento de producirse aquel" de modo que "la existencia o no del nexo causal o no entre el daño y la investigación deberá ser probada por el demandado", induce a acciones judiciales y obliga a prorrogar seguros más allá de los dos años posteriores al término total del ensayo para cumplir con lo señalado en el artículo 90, a diferencia de lo que establece el artículo 87: "los 2 años posteriores al término total del ensayo".

El plazo de 10 años para reclamar después de la manifestación del daño nos parece excesivo y la relación causal es extraordinariamente difícil de comprobar si se considera que el curso natural de las enfermedades se acompaña de complicaciones y daños propios y que, además, en este periodo el envejecimiento normal se asocia a eventos adversos propios de eventuales comorbilidades.

\section{Conclusiones}

$1^{\circ}$ En nuestra realidad nacional, una eventual aprobación de la propuesta de Reglamento para la aplicación de la Ley 20.850, como se presentó a nuestro estudio, nos hace predecir dificultades, judicialización y desincentivos a la investigación médica.

$2^{\circ}$ De no resultar factible una modificación en el Título V de la Ley, referido a la investigación clínica, se hace necesario que en el Reglamento se excluyan las responsabilidades por daños relacionables con la evolución de la enfermedad, o atribuibles al efecto del transcurso del tiempo en la persona, y por comorbilidades y eventos imprevisibles. 


\section{Bibliografía}

1.- RUBIO D M, SCHOENBAUM E E, LEE L S, SCHTEINGART D E, MARANTZ P R, ANDERSON $\mathrm{K}$ E, et al. Defining translational research: Implications for training. Acad Med 2010; 85: 470-5.

2.- INTERNATIONAL COMMITTEE OF MEDICAL JOURNAL EDITORS (ICMJE). Recommendations for the conduct, editing and publication of scholarly work in medical journals. Updated December 2016, disponible en www.icmje.org.

3.- REYES H. La relevancia del International Committee of Medical Journal Editors (ICMJE) para las publicaciones y la investigación médica. Rev Med Chile 2014; 142: 79-83.

4.- CLARKE L A, WRAITH J E, BECK M, KOLODNY E H, PASTORES G M, MUENZER J, et al. A multicenter, multinational, open-label extension study of the safety and efficacy of Aldurazyme ${ }^{\circledR}$ (Laronidase) in patients with mucopolysaccharidosis I. Pediatrics 2009; 123: 229-40. ClinicalTrials.gov NCT00146770.

5.- MUENZER J, WRAITH J E, BECK M, GIUGLIANI R, HARMATZ P, ENG C M, et al. A phase II/III clinical study of enzyme replacement therapy with idursulfase in mucopolysaccharidosis II (Hunter syndrome). Genet Med 2006; 8: 465-73.

6.- GIUGLIANI R, LAMPE C, GUFFON N, KETTERIDGE D, LEÃO-TELES E, et al. Natural history and galsulfase treatment in mucopolysaccharidosis VI (MPS VI, Maroteaux-Lamy syndrome)-10-year follow-up of patients who previously participated in an MPS VI Survey Study. Am J Med Genet A 2014; 164: 1953-64.

7.- RAIMANN E, CORNEJO V, ARIAS C, CABELLO J F, CASTRO G, FERNÁNDEZ E, et al. Evolución clínica de pacientes chilenos con tirosinemia tipo I tratados con 2-(2-nitro-4-trifluorometilbenzoil)- 1,3-ciclohexanediona (NTBC). Rev Med Chile 2012; 140: 169-75.

8.- MASUREL-PAULET A, POGGI-BACH J, ROLLAND M O, BERNARD O, GUFFON N, DOBBELAERE E, et al. NTBC treatment in tyrosinaemia type I: long-term outcome in French patients. J Inherit Metab Dis 2008; 31: 81-7.

9.- VITAL E M, EMERY P. Abatacept in the treatment of rheumatoid arthritis. Ther Clin Risk Manag 2006; 2 : 365-75.

10.- COHEN S B, EMERY P, GREENWALD M W, DOUGADOS M, FURIE R A, GENOVESE M C, et al. Rituximab for rheumatoid arthritis refractory to anti-tumor necrosis factor therapy: Results of a multicenter, randomized, double-blind, placebo-controlled, phase III trial evaluating primary efficacy and safety at twenty-four weeks. Arthritis Rheum 2006; 54: 2793 806.

11.- KAPPOS L, RADUE E-M, O'CONNOR P. Oral Fingolimod (FTY720) for relapsing multiple sclerosis.
N Engl J Med 2006;355:1124-1140. ClinicalTrials. gov NCT00333138 [core study] y NCT00235430 [extension].

12.- POLMAN C H, O'CONNOR P W, HAVRDOVA E, HUTCHINSON M, KAPPOS L, MILLER D H, et al. A Randomized, Placebo-Controlled Trial of Natalizumab for Relapsing Multiple Sclerosis. N Engl J Med 2006; 354: 899-910.

13.- ZIMRAN A, BRILL-ALMON E, CHERTKOFF R, PETAKOV M, BLANCO-FAVELA F, MUÑOZ E T, et al. Pivotal trial with plant cell-expressed recombinant glucocerebrosidase, taliglucerase alfa, a novel enzyme replacement therapy for Gaucher disease. Blood 2011; 118: 5767-73.

14.- WEINREB N J, GOLDBLATT J, VILLALOBOS J, CHARROW J, COLE J A, KERSTENETZKY M, et al. Long-term clinical outcomes in type 1 Gaucher disease following 10 years of imiglucerase treatment. J Inherit Metab Dis 2012; 36: 543-53.

15.- BANIKAZEMI M, BULTAS J, WALDEK S, WILCOX W R, WHITLEY C B, MCDONALD M, et al. Agalsidase-beta therapy for advanced Fabry disease: A randomized trial. Ann Intern Med 2007; 146 77-86.

16.- OLSCHEWSKI H, SIMONNEAU G, GALIÈ N, HIGENBOTTAM T, NAEIJE R, RUBIN L J, et al Inhaled iloprost for severe pulmonary hypertension. $\mathrm{N}$ Engl J Med 2002; 347: 322-9.

17.- GALIE N, RUBIN L J, HOEPER M M, JANSA P, ALHITI H, MEYER G M B, et al. Long-term pulmonary hemodynamic effects of ambrisentan in pulmonary arterial hypertension. Lancet 2008; 371: 2093-100. ClinicalTrials.gov NCT00091715.

18.- KLINGER J R, OUDIZ R J, SPENCE R, DESPAIN D, DUFTON C. Treatment of patients with mildly symptomatic pulmonary arterial hypertension with bosentan (EARLY study): a double-blind, randomised controlled trial. Am J Cardiol 2011; 108: 302-7.

19.- THE IMPACT-RSV STUDY GROUP. Palivizumab, a humanized respiratory syncytial virus monoclonal antibody, reduces hospitalization from respiratory syncytial virus infection in high-risk infants. The IMpact-RSV Study Group. Pediatrics 1998;102: 5317.

20.- SLAMON D, EIERMANN W, ROBERT N, PIENKOWSKI T, MARTIN M, PRESS M, et al. Adjuvant trastuzumab in HER2-positive breast cancer. N Engl J Med 2011; 365: 1273-83.

21.- DIGNASS A, VAN ASSCHE G, LINDSAY J O, LÉMANN M, SÖDERHOLM J, COLOMBEL J F, et al. The second European evidence-based consensus on the diagnosis and management of Crohn's disease: Current management. Journal of Crohn's and Colitis 2010; 4: 28-62

22.- PICKUP J, KEEN H. Continuous subcutaneous insulin infusion at 25 years. Evidence base for the expanding 
use of insulin pump therapy in type 1 diabetes. Diabetes Care 2002; 25: 593-8.

23.- SOFAER N. Reciprocity-based reasons for benefiting research participants: Most fail, the most plausible is problematic. Bioethics 2014; 28: 456-71.
24.- MIERES C. Las grietas de la Ley Ricarte Soto. Portafolio Salud. Junio 2015; 30-5.

25.- WHO International Clinical Trials Registry Platform (ICTRP). Disponible en www.who.int/ictrp/network/ primary/en/index.html 